through all these observations of the eyeball in health, in disease, and under the influence of drugs, there is on the one hand, a broad tendency towards obedience to definite physical and physiological laws, and on the other hand, an elf-like erratic element, which is extraordinarily puzzling, and which leaves on the mind the impression that there is some very important factor which we have hitherto been unable to identify or comprehend. May it be that this has to do with the anatomical and physical conditions which govern the escape of blood through the ocular tunics, or with the hydrodynamic conditions prevailing in the reservoirs into which the blood flows on its exit from the eye? If this question could be satisfactorily answered, it is possible that we should be nearer than we have ever hitherto been to the elucidation of yet another problem, viz., that of the elemental difference between simple and congestive glaucoma.

\title{
THE HALOS OF GLAUCOMA
}

BY

\section{R. H. Elliot, Lt.-Col. I.M.S. (Retd.) \\ LONDON}

AN endeavour to obtain precise information as to the nature and character of the halos seen by glaucomatous eyes, and under certain other conditions, speedily reveals the fact that there is but little accurate information to be obtained from the existing medical writings on the subject. Apparently few have thought it worthy of careful study, and the very great majority of ophthalmic surgeons are content to record the observation of coloured rings by a patient, without troubling to investigate the matter any further. The writer feels that this attitude is a mistaken one, and believes that valuable results may be obtained from a systematic study of these halos. In order to clear the ground it must first be stated that the coloured rings may be seen subjectively by an eye under a number of different conditions : (1) In the presence of the corneal oedema that accompanies a rise in ocular tension (the true glaucomatous halos). (2) The coloured rings seen by an eye which has been treated with African arrow-head poison or with silver nitrate are probably due to a transitory corneal oedema induced by the drugs, and therefore may be assumed to be closely comparable physically with those of true glaucoma. (3) When the surface of the eye is covered with thick mucus, faint coloured rings may be seen round a flame; these disappear at once on washing the eyes and are not of serious import. The writer believes them to be due to the presence in the mucus of yast numbers of tiny air bubbles. (4) When the pupils of certain patients are dilated by a mydriatic, somewhat faint, 
but quite definite, coloured rings can be constantly observed; Druault has suggested that these rings are to be attributed to diffraction of the light by the lens fibres at the level of the pupil (Morax). (5) In certain cases of nuclear cataract, distinct coloured halos can be observed; these are probably allied to Druault's diffraction phenomena. (6) The normal eye can, under suitable conditions, see coloured halos round bright lights in the dark. - These halos are fainter than those seen in glaucoma. (7) When lycopodium powder is dusted on a glass plate and a source of light is examined through the film so formed, definite colour rings are seen. The same is true of a steamed plate.

This is but a bare outline of a large and interesting subject, which the writer hopes to deal with very much more fully on a later occasion. His object in bringing the matter forward at the present time is to direct the attention of ophthalmologists to the extraordinary differences in the statements made by various observers as to the arrangement and the measurements of these halos. Quite recently he was fortunate enough to have the opportunity of examining a particularly intelligent and observant professional man, aged 36, who had been watching and attentively noting the nature of his halos before he sought advice for the relief of an intermittent subacute congestive glaucoma. The order of the colours was as follows: dark blue in the centre, then green, and yellow on the outside. He was examined in a dark room, at a distance of $11 \mathrm{ft} .7$ in. from a moderately bright frosted electric light, seen through a diaphragm 1 in. in diameter, and was first given a little time to gain a clear conception of the measurements of the different rings. The room was then dimly lit by ceiling lights, and the radii of the coloured rings were measured in turn on a long sheet of paper, one end of which was made to correspond with the vertical centre of the source of light under observation. The edges of the rings were then marked off on the paper. From these measurements the subtended angles were estimated in the usual way. The total measurement of the halo was found to be $8.5^{\circ}$.

The coloured halos seen through a steamed plate start with a measurement of about $9.5^{\circ}$ and rapidly contract as the small steam globules run into larger ones. This is in accord with what we know of the principles of diffraction, for the smaller the discs or globules that determine the halos, the larger the halos and vice versa. The lycopodium halos have a constant measurement (in the writer's experience of one sample of lycopodium) of $2^{\circ}$ to $2.5^{\circ}$. The physiological halos referred to under (6) above are likewise constant in measuring about $4^{\circ}$. On the other hand the figures given us for the glaucomatous halos are only $2^{\circ}$ to $2.5^{\circ}$, according to one observer and from $7^{\circ}$ to $8^{\circ}$, up to $10^{\alpha}$ to $12^{\circ}$, according to others. Putting, aside the first estimate as irreconcilable with all other experience 
the writer would suggest a simple explanation of the great difference in the results of the remaining observers. It is accepted that the cells which cause the diffraction phenonema belong to the corneal epithelium (and possibly to the endothelium). As these cells enlarge under the progress of oedema, the diameters of the halos they cause will correspondingly diminish; thus the size of the halo observed will probably differ according to the phase of the pathological process.

The writer ventures to place this note on record in the hope that others, who have the opportunity of making similar measurements on glaucomatous subjects, will do so, and will either publish their results, or be kind enough to communicate them to him. The distance at which the measurement is made is not of much importance, provided that it is sufficiently great to admit of the easy measurement of the radius of each circle. Knowing the distance from the eye to the observed source of light $(a)$ and the measurement of the halo from edge to edge (b), a simple formula enables us to calculate the angle $(\theta)$ required, viz., $\tan . \theta=\frac{a}{b}$; a reference to a logarithm table completes the calculation, and gives us the measurement of the angle in degrees. To put the matter approximately for those who desire to avoid all mathematical calculations. If the light is $10 \mathrm{ft}$. from the observing eye, the diameters of the halos will be as follows:-For $2^{\circ}, 4 \frac{1}{3}$ ins.; for $3^{\circ}, 6 \frac{1}{4}$ ins.; for $4^{\circ}, 8 \frac{1}{2}$ ins. ; for $5^{\circ}, 10 \frac{1}{2}$ ins. ; for $6^{\circ}, 12 \frac{1}{2}$ ins.; for $7^{\circ}, 14 \frac{3}{4}$ ins.; for $8^{\circ}, 17$ ins. ; for $9^{\circ}, 19$ ins.

\section{SAFETY IN OPHTHALMIC OPERATIONS}

BY

W. H. Simpson, M.B., Ch.B. (N.Z.), F.R.C.S.Ed.

LATE SENIOR HOUSE SURGEON, ROYAL LONDON OPHTHALMIC HOSPITAL (MOORFIELDS).

WHILE the elimination of suppuration in ophthalmic operations is probably an unattainable ideal, it is certain that the irreducible minimum has not yet been reached, and to that end our most earnest eftorts must be directed. No surgeon is perfect in his technique, and it appears reasonable that if one could practise the best points of many surgeons, one would be as nearly perfect as human error would allow. There is nothing original in the points set out below - they are a summary of lessons learned from observing the work of several British ophthalmic surgeons.

The remarks apply, of course, largely to the operation for extraction of cataract.

Preliminary examination, culture and treatment.-This is of first 